\title{
Tachistoscopic perception under head tilt
}

\author{
MICHAEL C. CORBALLIS, TERESA ANUZA, and LARRY BLAKE \\ McGill University, Montreal, Quebec, Canada
}

\begin{abstract}
Subjects attempted to identify singly presented letters, flashed briefly at six different locations equidistant from fixation. The locations were at $1,3,5,7,9$, and 11 o'clock on an imaginary circle. The subjects performed both with their heads upright or tilted $60^{\circ}$ (i.e., through 2 and 8 o'clock or through 10 and 4 o'clock). In Experiment 1, the letters were projected in a gravitationally fixed rectangular surround, and accuracy of report depended largely on the locations of the letters in gravitational coordinates, although there was clear evidence of a retinal influence as well. In Experiment 2, the surround was circular and accuracy depended principally on retinal location, although there was also a gravitational influence. Analysis of individual data suggested that, when their heads were tilted, subjects generally adopted a reference frame which lay between the retinal and gravitational frames, closer to one than the other but typically coinciding with neither. We conclude that the subjective reference frame does not depend on a poststimulus correction, but rather that stimulus information is incorporated directly into the subjective frame. The data also suggested that lateral asymmetries depend on a perceptual rather than a retinal interpretation of left and right visual fields.
\end{abstract}

It has been noted that the experience of visual space bears little direct relation to the retinal image (e.g., Gibson, 1966; Turvey, 1977). For instance, the perceptual world remains stable when we move our eyes or tilt our heads. Nevertheless, there cannot, of course, be a complete dissociation between the visual percept and the retinal image, since visual information about the world is necessarily channeled through the retina and the retinocortical pathways. It remains an empirical question as to which aspects of visual spatial perception are tied to retinal coordinates and which to gravitational coordinates. In some cases, too, perceptual coordinates may correspond with neither the retinal nor the gravitational ones.

Previous evidence suggests that the coordinates underlying spatial perceptual effects may depend partly on the nature of the stimuli and partly on the task, although these two factors are often confounded in comparisons across different experiments. Memory for novel figures (Rock \& Heimer, 1957), speeded identification of line slopes (Attneave \& Olson, 1967), and the mental rotation of alphanumeric characters (Corballis, Zbrodoff, \& Roldan, 1976) all seem to be accomplished with reference to gravitational rather than retinal coordinates when subjects tilt their heads-although in the last two examples the choice of reference frame is to some extent under voluntary control (Attneave \& Reid, 1968; Corballis, Nagourney, Shetzer, \& Stefanatos,

Supported by a grant from the National Research Council of Canada. M.C. Corballis is now at the Department of Psychology, University of Auckland, Private Bag, Auckland, New Zealand.
1978). The perception of symmetry provides an ambiguous case; Rock and Leaman (1963) found that the influence of symmetry on judgments of perceptual similarity was tied to gravitational rather than retinal coordinates, but Corballis and Roldan (1975) reported a strong retinal influence underlying speeded judgments of whether or not dot patterns were symmetrical about a line. Olson and Attneave (1970) found that perceptual grouping seemed to be based more on retinal than on gravitational coordinates. Rock (1973) suggested that the retinal factor prevails in the perception of faces and in the perception of cursive script, largely on the grounds that these stimuli are extremely difficult to identify when upside down relative to the observer.

Rock (1973) went on to argue that visual stimuli are initially registered on retinal coordinates, but that the retinally based percept is "more or less immediately" (p. 49) superceded by one that is corrected for head tilt or for tilt of the stimuli. There is, indeed, evidence that observers cannot adjust their perceptual reference frames in anticipation of a pattern of unknown identity but known tilt, suggesting that correction for a tilted stimulus can take place only after it has appeared (Cooper \& Shepard, 1973; Corballis \& Roldan, 1975). But this does not seem to be true of the correction for head tilt. We have already cited evidence that subjects with their heads tilted can make speeded perceptual judgments within a gravitational rather than a retinal framework (Attneave \& Olson, 1967; Corballis et al., 1976). These results call into question Rock's notion that there is a poststimulus correction for head tilt. If there is such a correction, it must be "more" rather 
than "less" immediate, and it almost certainly does not involve mental rotation, as Rock $(1973$, p. 74$)$ later suggested (cf. Corballis et al., 1976). We think it more likely that the correction for head tilt can actually precede presentation of the stimulus.

The present experiments are a preliminary attempt to determine the reference frame underlying the accuracy with which observers can report tachistoscopically presented information when their heads are tilted. Specifically, we wished to determine whether the accuracy with which a subject can report a tachistoscopically presented letter depends on the retinal location of the letter, on its location in gravitational coordinates, or perhaps on its location within a subjective coordinate system lying between the retinal and gravitational systems. With tachistoscopic presentation, there would presumably be a severe limitation on the degree of poststimulus correction for head tilt, so that the empirically determined reference frame might be considered a reasonable approximation to the frame within which the stimulus is initially registered.

Earlier experiments on the coordinate system underlying spatial effects in the identification of tachistoscopically presented material have had to do with eye movements, not head tilt. Davidson, Fox, and Dick (1973) found that if a saccade intervenes between presentation of an array of elements and a mask, the element that is masked is that which occupies the same retinal location as the mask, even though the two occupy different perceived locations. On the other hand, White (1976) reported that if a pursuit eye movement intervenes between the array and the mask, the element that is masked is that which occupies the same actual location in space (or close to it) as the mask, even though the mask and target occurred in different retinal locations. These results suggest that the iconic representation is not unitary; in some respects it seems tied to the coordinates of perceived space, in other respects to the coordinates of retinal space (see Turvey, 1977).

The question of whether accuracy of report depends on retinal or perceived location also bears on the nature of lateral asymmetries in the identification of tachistoscopically presented items. Verbal items such as letters or words are typically identified more accurately if flashed in the right than in the left visual field, at least among righthanded subjects (e.g., Mishkin \& Forgays, 1952; White, 1969), while nonverbal items such as faces (Geffen, Bradshaw, \& Wallace, 1971) or meaningless spatial configuration (Kimura, 1969) tend to elicit a left-field advantage. These effects are commonly attributed to the fact that information in the right visual field is projected to the left cerebral hemisphere while left-field information is projected to the right cerebral hemisphere, so that field differences reflect the differential hemispherical specializations (e.g., Bryden, 1965; Kimura, 1966). But this account is based on the anatomy of the visual pathways, and implies a retinal interpretation of left and right visual fields. There are hints that laterality effects are best interpreted as perceptual rather than anatomical. For instance, Kinsbourne (1975) found that if subjects were given a concurrent verbal task, they were more accurate in detecting a gap on the right side of a briefly flashed square than in detecting a gap on the left side. However, this asymmetry did not depend on which visual field the square was in; it was the side of the square, left or right, which was more important, regardless of whether the square was wholly to the left of fixation, wholly to the right, or symmetrical about fixation. Laterality effects comparable to those in tachistoscopic perception have also been reported in auditory perception, principally through the use of dichotic-listening techniques (e.g., Kimura, 1961, 1967). Morais and Bertelson (1975) have reported evidence that the right-sided advantage in the processing of dichotically presented speech sounds depends on the perception of whether a sound is to the left or right rather than which ear receives the sound. Again, this suggests that the asymmetry may be perceptual rather than anatomical.

Part of our aim in the experiments to be reported below was to develop another technique for distinguishing between the anatomical and perceptual bases of asymmetry in tachistoscopic perception. Although it is now a fairly commonplace observation that "higher-order" processes such as attention or set may influence the degree or even direction of visual laterality (e.g., Hellige, 1978; Kinsbourne, 1973), this does not necessarily imply that laterality is decoupled from the anatomical links between retina and cortex. That is, higher-order activation of a given hemisphere may induce an asymmetry, but this asymmetry may still be manifest in terms of retinal location. To our knowledge, the only previous experiment which does suggest that visual laterality may be decoupled from a retinal reference frame is Kinsbourne's (1975) experiment on gap detection, described above. The investigation of laterality effects under head tilt seem to provide an alternative method for decoupling the perceptual from the anatomical reference frame.

An overview of the experimental approach is as follows: Letters were projected singly at different locations which can be identified with reference to an imaginary clockface. On gravitational coordinates, these locations were at $1,3,5,7,9$, and 11 o'clock (see Figure 1). Thus, with the head upright, 1, 3 , and 5 o'clock are in the right visual field, while 7,9 , and 11 o'clock are in the left visual field. 


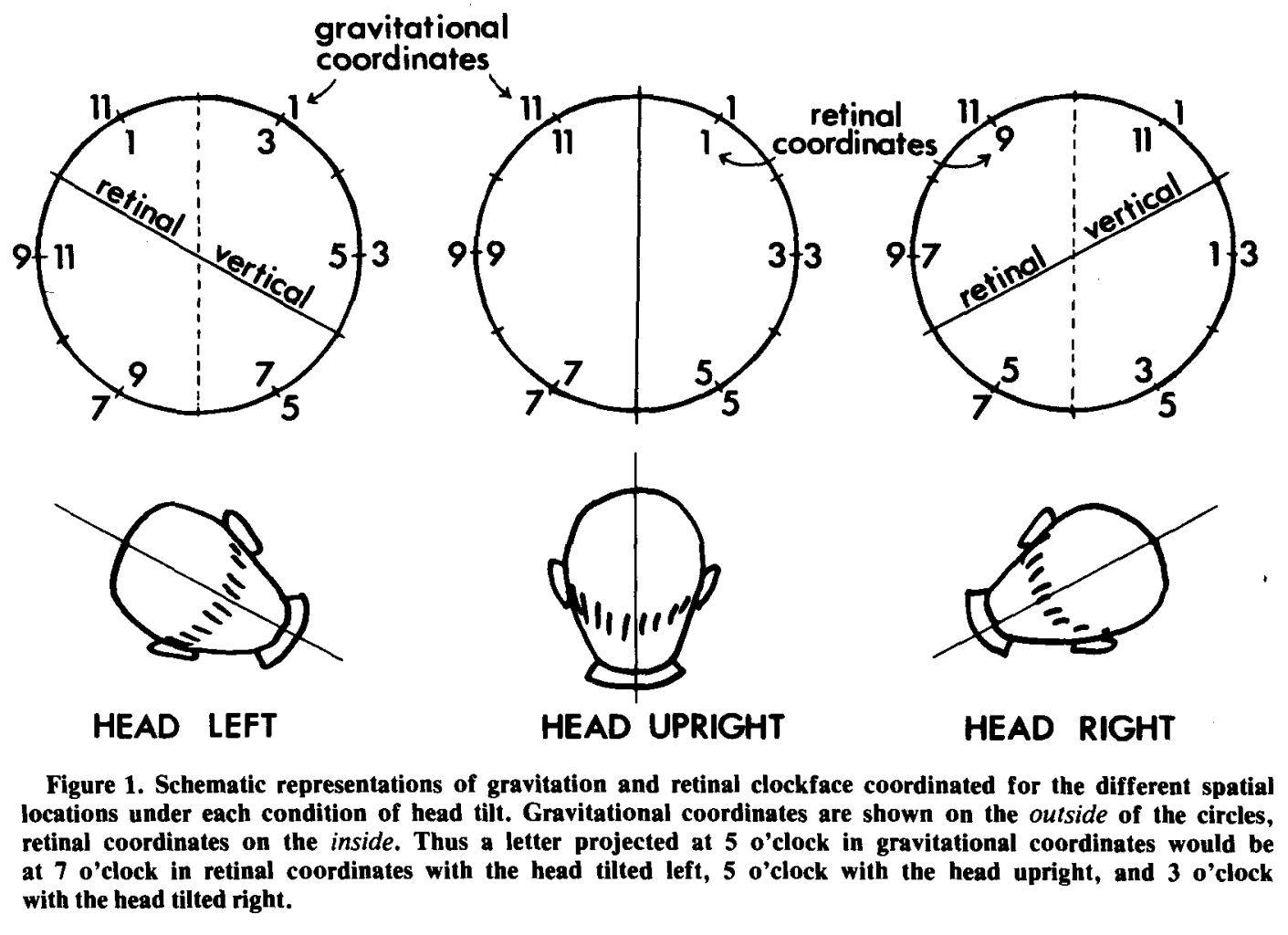

With the head tilted to the right such that the vertical retinal meridian is at $60^{\circ}$ to the gravitational vertical (that is, running through 8 and 2 o'clock), the gravitational positions $1,3,5,7,9$, and 11 o'clock correspond to retinal positiona $11,1,3$, 5,7 , and 9 o'clock, respectively. Similarly, with the head tilted to the left, the corresponding retinal coordinates are $3,5,7,9,11$, and 1 o'clock, respectively. The subjects attempted to identify the letters both with their heads upright and with their heads tilted, either to the left or to the right. Our primary aim was to determine whether the pattern of accuracy scores as a function of position shifted when the subjects tilted their heads. That is, was the pattern more nearly invariant across head-tilt conditions when position was measured in gravitational coordinates or when it was measured in retinal coordinates?

The letters were also displayed in two different angular orientations at each location: they were either upright in gravitational space or rotated $60^{\circ}$ in the same direction as the subject's head was tilted. We were thus able to determine whether accuracy was higher when the letters were upright in gravitational coordinates or when they were upright in retinal coordinates. We were also able to tell whether the reference frame underlying position effects was dissociable from that underlying orientation effects.

\section{EXPERIMENT 1}

\section{Method}

Subjects. The subjects were 12 men and 12 women, drawn from undergraduate classes. All professed to be right handed and to possess normal or corrected-to-normal vision.

Stimuli and Apparatus. The stimuli were presented in a threefield Gerbrands tachistoscope. The eyepiece was modified so that it could be rotated either clockwise or counterclockwise. For head-tilt conditions, the angle was set at $66^{\circ}$ from the upright, and the subjects sat with their heads also tilted at $66^{\circ}$ and snug against the eyepiece. Assuming that there is counterrolling of the eyes of about $10 \%$ (Miller, 1962), this meant that the subjects' eyes were rotated $60^{\circ}$ from the upright position when their heads were tilted.

The stimuli consisted of the letters $A, H, M, T, U, V, W$, and $\mathrm{Y}$, which were presented in six different spatial locations. Letters symmetrical about the vertical axis were chosen to avoid bias in favor of one or the other visual field. They were made from black Letraset (No. 520 Futura), affixed to white cards; in height, they subtended a visual angle of $0^{\circ} 30^{\prime}$. The locations were such that the center of each letter was a visual angle of $3^{\circ} 01^{\prime}$ from a central fixation point; on an imaginary clockface, the locations were at $1,3,5,7,9$, and 11 o'clock-or at $30^{\circ}, 90^{\circ}$, $150^{\circ}, 210^{\circ}, 270^{\circ}$, and $330^{\circ}$ clockwise from the 12 o'clock position (see Figure 1). For half of the trials, the letters were presented in the normal upright position, while for the other half, they were tilted $60^{\circ}$ in the direction in which the subject's head was tilted. The stimuli appeared in a rectangular visual field measuring $8^{\circ} 30^{\prime}$ wide $\times 6^{\circ} 05^{\prime}$ high.

For $90 \mathrm{msec}$ immediately prior to the stimulus presentation, the fixation point was replaced by either a solid black square or a solid black circle. The diameter of the circle and the width of the square both subtended $0^{\circ} 17^{\prime}$. The square was either upright or tilted $60^{\circ}$ to the left or right. The subjects 
were required to report whether this central stimulus was a circle or a square before reporting the letter; this was to ensure central fixation.

Procedure. In a preliminary session, each subject was given blocks of 15 trials with his/her head upright and with upright letters, beginning with an exposure duration of $40 \mathrm{msec}$. The duration was altered over successive blocks until the subject's accuracy over the block was close to $50 \%$ (i.e., 7 or 8 out of 15).

Once the criterion was reached, the exposure duration was fixed for the remainder of the trials. The exposure durations ranged from 6 to $48 \mathrm{msec}$, with a mean of $27.0 \mathrm{msec}$. There were four experimental sessions. In the first two sessions, the letters were always upright. Each letter was presented once in each position, making a total of 48 trials per session. In one session, the subject performed with the head upright, in the other his/her head was tilted; the order was counterbalanced between subjects. Half of the subjects tilted their heads to the left, half to the right. The entire procedure was repeated over the third and fourth sessions, except that the letters were tilted in the direction of head tilt. The letters were tilted during both sessions, even when the subject's head was upright. In each of the subgroups formed by the direction of head tilt and the counterbalancing of head position, there were equal numbers (three) of men and women.

The stimulus cards were ordered randomly. The choice of geometric form (circle or square) was also determined randomly. The square was always aligned with the head. If a subject failed to identify the form, the trial was discounted and the stimulus was repeated later in the sequence.

Subjects were informed of the set of letters in advance, and were thus able to guess if not sure of a letter on any given trial.

\section{Results}

Analysis of variance was carried out on the number of letters identified at each position; the independent variables were sex, order of receiving head-tilt conditions, head tilt (upright vs. tilted), direction of head tilt (left vs. right), letter position, and letter tilt (upright vs. tilted).

Effects of letter tilt. Tilted letters were identified significantly ${ }^{1}$ less often than upright letters $[F(1,16)$ $=45.13]$. This effect may have been due partly to fatigue, since tilted letters were always presented in the third and fourth sessions-a design flaw which is corrected in the following experiment. There was also a significant interaction between head tilt and letter tilt $[F(1,16)=21.69]$. When the letters were upright, they were identified more often when the head was also upright than when it was tilted (mean scores out of eight were 4.597 and 3.931 , respectively). When the letters were tilted, however, they were better recognized when the head was also tilted than when the head was upright (means of 3.299 and 2.743 , respectively)-letter tilt was always in conformity with head tilt. These results, although contaminated by a possible fatigue effect, suggest that both gravitational and retinal letter tilt produce decrements in identification, but that gravitational tilt has the stronger influence; estimated variance components (see Vaughan \& Corballis, 1969) were .7555 and .3562 , respectively.
There was also a significant interaction between letter tilt and sex $[F(1,16)=6.51]$. The men and the women identified about the same number of upright letters ( means of 4.26 and 4.27 , respectively), but the women identified more tilted letters than the men did (3.50 and 2.54, respectively). There were no other significant interactions involving either letter tilt or sex.

Position effects. The effect of letter position was assessed according to both gravitational and retinal coordinates. Retinal coordinates were based on the assumption that the subjects' retinas were tilted $60^{\circ}$ under the head-tilt conditions (see Figure 1). In both cases, the main effect of letter position was highly significant $[F(5,80)=35.47$ for gravitational coordinates and $F(5,80)=28.35$ for retinal coordinates]. The estimated variance components were .8344 and .6814 , respectively. In determining the reference frame within which position effects are manifest, however, it is more to the point to consider the influence of head tilt on position effects.

Position effects varied with head tilt in both gravitational and retinal coordinates. In gravitational coordinates there were significant interactions between position and head tilt $[F(5,80)=3.59]$, position and direction of head tilt $[\mathrm{F}(5,80)=14.52]$, and position, head tilt, and direction of head tilt $[F(5,80)=4.23]$. In retinal coordinates, these interactions were also significant $[\mathrm{F}(5,80)=8.33, \mathrm{~F}(5,80)$ $=6.26$, and $F(5,80)=15.21$, respectively]. In order to probe further the nature of the effects underlying these interactions, we computed the two-way interactions between letter position and head tilt separately for each direction of head tilt, in both gravitational and retinal coordinates.

For those subjects whose heads were tilted left, the interaction between head tilt and letter position was only barely significant when position was defined gravitationally $[F(5.80)=2.99]$. This is insignificant according to the conservative degrees of freedom $(1,16)$ recommended by Winer $(1971)$ for testing repeated-measurement effects. It was highly significant when position was defined retinally $[F(5,80)=14.03]$. The estimated variance components were .1851 and 1.1704 , respectively, indicating that position effects were more nearly invariant with respect to head tilt when a gravitational reference frame is assumed. For those subjects whose heads were tilted right, the interaction was significant on gravitational coordinates $[F(5,80)$ $=4.83]$ and also significant on retinal coordinates $[F(5,80)=9.51]$. The estimated variance components were .3557 and .7646 , respectively. Again, therefore, position effects were more nearly invariant according to the gravitational coordinate system, although there was clear evidence here of a retinal influence as well. 
It was fairly clear from the data, however, that there were individual differences in the location of the reference frame underlying the position effects. In order to assess the reference frame adopted by each individual, cross correlations were computed between the position functions obtained with the head upright and those obtained with the head tilted. Since there was no indication either from the analysis of variance or from inspection of the data that the effect of position depended upon letter tilt, the scores for each position were summed over upright and tilted letters. Six different correlations were then computed for each subject. In order to understand how this was done, the reader may find it helpful to refer to Figure 1; in what follows, all positions are specified in gravitational coordinates. First, the positions were matched according to their actual (gravitational) locations; that is, the accuracy scores for letters at $1,3,5,7,9$, and 11 o'clock obtained with the head upright were correlated with those at $1,3,5,7,9$, and 11 o'clock, respectively, obtained with the head tilted. The scores under the head-tilt condition were then successively rotated counterclockwise while those under the head-upright condition were held constant, and the correlation was computed in each case. Thus the head-upright scores at 1, 3, 5, 7, 9, and 11 o'clock were correlated with the head-tilt scores at $3,5,7,9$, 11 , and 1 o'clock, respectively, then with the headtilt scores at 5, 7, 9, 11, 1, and 3 o'clock, respectively, and so on. Notice that the very first match is always a gravitational one, the second is a retinal match for subjects who tilted their heads to the left, and the sixth is a retinal match for subects who tilted their heads to the right. The degree of positive correlation for any given match is a measure of the degree of the invariance across position effects when measured on the reference frame specified by that match. Thus a subject was designated "gravitational" if the highest positive correlation occurred when the locations were gravitationally matched, "retinal" if the highest positive correlation occurred when the locations were retinally matched, and "anomalous" otherwise. Among the 12 subjects who tilted their heads to the left, 8 were gravitational, 1 retinal, and 3 anomalous. Among the 12 who tilted their heads to the right, 6 were gravitational, 5 retinal, and 1 anomalous. Thus for the majority of subjects, 14 out of 24 , the effects of position on accuracy were dependent upon gravitational rather than retinal position.

For the 14 "gravitational" subjects, the mean correlation for the gravitational match was .629 , while for the six "retinal" subjects, the mean retinal correlation was .759. It was also of interest to enquire whether there was any retinal influence among the gravitational subjects and any gravitational influence among the retinal subjects. Such influences would be revealed by asymmetries in the cross correlation functions about the peak correlation. Thus, 12 of the 14 gravitational subjects revealed a higher correlation (mean for the group $=.011$ ) for the retinal match than for the match corresponding to $60^{\circ}$ rotation in the direction opposite to the retinal match (mean for the group $=-.387$ ), which differs significantly from chance according to a sign test. All six retinal subjects revealed higher positive correlations for the gravitational match (mean $=.305$ ) than for the match corresponding to $60^{\circ}$ in the opposite direction (mean $=-.347$ ), which again differs significantly from chance. This analysis suggests that the reference frames within which position effects occurred were not aligned precisely with either gravitational or retinal coordinates, even in individual cases, but typically lay somewhere in between.

Table 1 shows the accuracy scores as a function of gravitational coordinates for the 14 subjects classified as "gravitational," while Table 2 shows accuracy as a function of retinal coordinates for the subjects classified as "retinal."

As noted earlier, there was no evidence from the analysis of variance for sex differences in relation to head-tilt effects. Moreover, exactly half of the gravitational subjects were male, as were exactly half of the retinal subjects. Those who tilted their heads to the left included more who were classified as gravitational than did those who tilted their heads to the right; disregarding the subjects classified as anomalous, however, the contingency between direction of head tilt and classification as gravitational or retinal was not significant according to Fisher's exact test $(\mathrm{p}=.238)$.

As the tables indicate, the pattern of position effects was such that those letters aligned horizontally with fixation (i.e., those displayed at 3 and 9 o'clock) were generally reported more accurately than those above and below fixation. On gravitational coordinates, the letters above and below fixation were closer to the borders of the rectangular surround than those aligned horizontally with fixation, so that one might be tempted to attribute the pattern of position effects partly to masking or metacontrast effects between the letters and the surround. However, Table 2 reveals essentially the

Table 1

Mean Accuracy as a Function of Letter Position in Gravitational Coordinates in Experiment 1, for Those 14 Subjects Classified as "Gravitational"

\begin{tabular}{lcccccc}
\hline & \multicolumn{6}{c}{ Letter Position on Clockface } \\
\cline { 2 - 7 } Hosition & 1 & 3 & 5 & 7 & 9 & 11 \\
\hline Upright & 3.357 & 5.107 & 2.964 & 2.536 & 5.250 & 3.179 \\
Tilted & 3.250 & 4.393 & 2.750 & 2.893 & 4.964 & 3.607 \\
\hline
\end{tabular}


Table 2

Mean Accuracy as a Function of Letter Position in Retinal Coordinates in Experiment 1, for Those 6 Subjects Classified as "Retinal"

\begin{tabular}{lcccccc}
\hline & \multicolumn{6}{c}{ Letter Position on Clockface } \\
\cline { 2 - 7 } Head & 1 & 3 & 5 & 7 & 9 & 11 \\
\hline Position & 1 & 3 & 5.650 & 1.583 & 4.500 & 3.25 \\
Upright & 3.667 & 4.667 & 2.250 & \\
Tilted & 4.500 & 4.000 & 1.417 & 1.917 & 4.750 & 3.417 \\
\hline
\end{tabular}

same pattern on retinal coordinates for those subjects classified as retinal; for this group, the interaction between head tilt and position was not significant on retinal coordinates $[F(5,25)=2.08]$, suggesting that position effects were indeed approximately invariant. Some degree of gravitational influence can be detected, however; that accuracy was slightly higher for letters at 1 o'clock than for those at 3 o'clock can be attributed to the fact that five of the six subjects in this group tilted their heads to the right. But accuracy was highest for letters at 9 o'clock on retinal coordinates, even though this position was at 11 o'clock on gravitational coordinates for all but one of the subjects and was therefore close to the top of the border. Thus, position effects do not seem to be attributable to metacontrast or masking; they appear to be a function of subjective space rather than physical space.

Laterality effects. Finally, we examined differences between the visual fields. The position effect can be broken down into three orthogonal components, left vs. right visual field, elevation (top pair vs. middle pair vs. bottom pair), and the interaction between fields and elevation. When the subjects viewed with their heads upright, letters in the right visual field were identified somewhat more accurately than those in the left, but the difference was not significant $[F(1,80)=2.82]$. The effect of position was largely due to elevation $[F(2,80)=$ 70.50], which did not interact significantly with visual field $[F(2,80)<1]$. Because the effect of visual field was not reliable even under the head-upright condition, there seemed no way to enquire from the grouped data under the headtilt conditions whether any laterality effects were manifest in gravitational or in retinal coordinates. We therefore attempted an individual analysis. Lateralization scores were computed for each subject by subtracting the number of letters identified in the left visual field from the number identified in the right. When the fields were defined gravitationally, the correlation between the lateralization scores under the head-tilt and headupright condition was .488 , which is significantly larger than zero $[\mathrm{t}(22)=2.62]$. But when the fields were defined retinally, the correlation was only .229 , which does not differ significantly from zero [t(22)
$=1.10]$. However, these two (correlated) correlations do not differ significantly from each other $[t(21)$ $=1.40 \mathrm{]}$, so this analysis provides only weak evidence that lateralization effects are gravitational rather than retinal.

\section{Discussion}

In this experiment, the reference frame within which position effects were manifest was more nearly invariant on gravitational than on retinal coordinates, at least among the majority of subjects, although there was clear evidence for at least some retinal influence. This gravitational effect may have been at least partly due to the rectangular surround, which remained fixed in gravitational coordinates even when the subjects tilted their heads. Based on analysis of position effects among the minority of subjects whose position effects were more nearly invariant on retinal than on gravitational coordinates, however, we concluded that the effect of the surround was not merely one of metacontrast or simultaneous masking. Thus, if the surround had any influence at all, it probably served to establish a subjective reference frame that was gravitational rather than retinal for most subjects. In order to assess this possibility, the rectangular surround was replaced by a circular surround in Experiment 2 .

Experiment 1 also failed to elicit a reliable difference between visual fields, so the question of whether visual lateralization is manifest in gravitation or in retinal coordinates was not satisfactorily resolved. It is possible that the subsidiary fixation task, in which the subjects were required to identify a central stimulus as a circle or a square, acted to induce a nonverbal set in some subjects which may have counteracted any potential rightfield advantage. Indeed, Kershner, Thomae, and Callaway (1977) have recently demonstrated just such an effect; they found that a nonverbal fixation task induced a left-field advantage in the report of tachistoscopically presented digits by young children, even though the same children displayed a right-field advantage if the fixation task was verbal. In the following experiment, therefore, we replaced the circle and square by the digits 0 and 8 .

\section{EXPERIMENT 2}

\section{Method}

Subjects. The subjects were 16 men and 16 women, all professing to be right-handed and to possess normal or corrected vision. They were drawn from undergraduate classes.

Stimuli and Apparatus. The stimuli and apparatus were the same as in Experiment 1, except for the following modifications: The stimulus set was reduced to the four letters $A, H, M$, and $\mathrm{T}$. The square and circle used as fixation stimuli in Experiment 1 , were replaced by the digits 0 and 8 , each of which subtended a maximum of $0^{\circ} 17^{\prime}$ at the subject's eyes. Finally, the rectangular surround in the tachistoscope was replaced by a 
circular surround of radius $3^{\circ} 8^{\prime}$, and the distance of each letter from the fixation point was reduced to $2^{\circ} 21^{\prime}$.

Procedure. The procedure was the same as in Experiment 1, with the following modifications: First, each stimulus was presented twice per session, so that the number of trials was the same as in Experiment 1. Secondly, the order of head-tilt conditions and of letter-tilt conditions were both counterbalanced. Half of the subjects received the upright letters followed by the tilted letters, and half received the tilted followed by the upright. Within these conditions, half of the subjects performed first with their heads tilted and then with their heads upright, and half worked in the reverse order. There were therefore four different orders of head-tilt and letter-tilt conditions. Other betweensubject factors were sex and direction of head tilt, which were crossed with each other and with order. There were two subjects within each of the 16 groups so formed. Each subject received a total of 192 trials, consisting of the four letters presented twice in each of six positions over four sessions, except that if a subject failed to report the fixation digit correctly that trial was repeated.

The exposure durations for the stimuli ranged from 7 to $23 \mathrm{msec}$, with a mean of $12.2 \mathrm{msec}$. These were somewhat shorter than in Experiment 1, perhaps because of the reduced size of the stimulus set.

\section{Results}

Analysis of variance was carried out on the number of letters identified at each position.

Effects of letter tilt. Tilted letters were again identified less accurately than upright letters, $[F(1,16)=7.20]$, suggesting that the effect of letter tilt in Experiment 1 was not due entirely to fatigue. Moreover, there was again a significant interaction between head tilt and letter tilt $[F(1,16)=22.92]$, which is shown in Table 3 . This can be interpreted to mean that accuracy was decreased by retinal tilt as well as by gravitational tilt. Indeed, retinal tilt seems to have had a stronger influence than gravitational tilt; the estimated variance components were .3282 and .0501 , respectively. In this respect, the results are the opposite of those obtained in Experiment 1.

There was no interaction between sex and letter tilt in this Experiment $[\mathrm{F}(1,16)=.01]$. Moreover, letter till did not interact significantly with letter position, head tilt, or direction of head tilt, or with any combination of these factors. However, the effect of letter tilt did depend on the reference frame adopted by the subjects, where the reference frame was determined with respect to the effect of letter position. This is discussed below.

Position effects. The effect of letter position was again assessed according to both gravitational and

Table 3

Mean Accuracy as a Function of Letter Tilt and Head Tilt in Experiment 2

\begin{tabular}{ccc} 
Head & \multicolumn{2}{c}{ Letter Relative to Gravity } \\
\cline { 2 - 3 } Position & Upright & Tilted \\
\hline Upright & 4.365 & 3.438 \\
Tilted & 3.625 & 3.870 \\
\hline
\end{tabular}

retinal coordinates. The main effect of position was significant in both cases $[F(5,80)=15.65$ for gravitational coordinates and $F(5,80)=21.77$ for retinal coordinates]. The estimated variance components were .3375 and .6025 , respectively.

Position effects were again influenced by head tilt on both gravitational and retinal coordinates. On gravitational coordinates, there were significant interactions between position and head tilt $[\mathrm{F}(5,80)$ $=11.92]$, position and direction of head tilt $[F(5,80)$ $=39.44]$, position, head tilt, and direction of head tilt $[F(5,80)=4.25]$, and position, head tilt, direction of head tilt, and sex $[F(5,80)=4.11]$ although the last two interactions are not significant according to conservative degrees of freedom $(1,16)$. On retinal coordinates, there were significant interactions between position and head tilt $[F(5,80)=$ $11.53]$, position and direction of head tilt $[F(5,80)$ $=23.79]$, position, direction of head tilt, and sex $[F(5,80)=5.06]$, and position, head tilt, and direction of head tilt $[F(5,80)=8.61]$. To clarify these effects, we computed the simple interactions between position and head tilt separately for men and women within each direction of head tilt, in both gravitional and retinal coordinates.

For the sub-group of eight men who tilted their heads to the left, the interaction between head tilt and position was significant when position was measured on gravitational coordinates $[F(5,80)=$ $6.50]$, but not significant on retinal coordinates $[F(5,80)=1.01]$. The estimated variance components were .7495 and .0012 , respectively. For this subgroup, therefore, position effects were manifest in retinal rather than in gravitational coordinates. A crosscorrelational analysis on individual scores, carried out as in Experiment 1, revealed that five subjects were classified as retinal, one as gravitational, and two as anomalous.

For the women who tilted their heads to the left, the interaction was again significant on gravitational coordinates $[F(5,80)=5.90]$ but not on retinal coordinates $[\mathrm{F}(5,80)=2.23]$. The estimated variance components were .6680 and .1083 . Again, therefore, position effects were manifest in retinal rather than in gravitational coordinates. According to the crosscorrelational analysis, five subjects were classified as retinal, one as gravitational, and two as anomalous.

For the eight men who tilted their heads to the right, the interaction was significant both on gravitational coordinates $[\mathrm{F}(5,80)=4.40]$ and on retinal coordinates $[F(5,80)=9.43]$. For this group taken as a whole, therefore, position effects were more nearly invariant on gravitational than on retinal coordinates; the estimated variance components were .4630 and .7444 . However, the crosscorrelational analysis produced an even split, with four subjects classified as retinal and four as gravitational. 
For the eight women who tilted their heads to the right, the interaction was again significant both on gravitational coordinates $[F(5,80)=5.30]$ and on retinal coordinates $[F(5,80)=11.58]$. The estimated variance components were .5854 and .9348 . Crosscorrelational analysis revealed that two were retinal, three were gravitational, two had equal correlations in gravitational and retinal coordinates, and one was anomalous.

Overall, therefore, position effects were more nearly invariant on retinal than on gravitational coordinates, but there was clear evidence for a gravitational influence. Sixteen subjects were classified as retinal (mean correlation $=.753$ ), 9 as gravitational (mean correlation $=.719$ ), 2 as equally split between gravitational and retinal (correlations of .505 and .678), and 4 as anomalous. Among the 16 classified as retinal, 13 revealed a higher correlation for the gravitational match (mean for the group $=.322$ ) than for the match corresponding to $60^{\circ}$ rotation in the other directon from the retinal match (mean $=-.084$ ), which is significant according to a sign test. Among the 9 subjects classified as gravitational, 5 revealed a higher correlation for the gravitational match (mean for the group $=.279$ ) than for the match corresponding to $60^{\circ}$ rotation in the opposite direction (mean $=.052$ ), but this is not significant according to a sign test. Table 4 shows the position effects on retinal coordinates for those subjects classified as retinal, and Table 5 shows the position effects on gravitational coordinates for those classified as gravitational.

The retinal and gravitational subjects differed with respect to the influence of letter tilt on identification. For the 16 subjects classed as retinal, tilt of the letters had a significant effect when measured in retinal coordinates $[F(1,16)=11.17]$, but not when measured in gravitational coordinates $[F(1,16)=$ 2.22]. Retinal tilt reduced mean accuracy from 3.896 to 3.156 , while gravitational tilt reduced it from 3.521 to 3.354. For the 9 subjects classed as gravitational, the results were the converse: tilt of the letters had a significant effect when measured in gravitational coordinates $[F(1,8)=5.73]$ but not when measured in retinal coordinates $[F(1,8)=$ 1.65]. Gravitational tilt reduced accuracy from 4.296 to 3.935 , while retinal tilt reduced it from 4.241 to 3.991 . These results indicate that the classification

Table 4

Mean Accuracy as a Function of Letter Position in Retinal Coordinates in Experiment 2, for Those 16 Subjects Classified as "Retinal"

\begin{tabular}{lcccccc}
\hline & \multicolumn{6}{c}{ Letter Position on Clockface } \\
\cline { 2 - 7 } $\begin{array}{c}\text { Head } \\
\text { Position }\end{array}$ & 1 & 3 & 5 & 7 & 9 & 11 \\
\hline Upright & 3.375 & 4.781 & 3.344 & 2.656 & 4.188 & 2.780 \\
Tilted & 3.688 & 5.000 & 3.219 & 2.188 & 3.750 & 3.344 \\
\hline
\end{tabular}

Table 5

Mean Accuracy as a Function of Letter Position in Gravitational Coordinates in Experiment 2, for Those 9 Subjects Cassified as "Gravitational"

\begin{tabular}{lcccccc}
\hline & \multicolumn{6}{c}{ Letter Position on Clockface } \\
\cline { 2 - 7 } $\begin{array}{c}\text { Head } \\
\text { Position }\end{array}$ & 1 & 3 & 5 & 7 & 9 & 11 \\
\hline Upright & 3.056 & 5.611 & 5.278 & 4.444 & 4.889 & 2.722 \\
Tilted & 3.278 & 4.556 & 5.556 & 4.222 & 4.000 & 2.500 \\
\hline
\end{tabular}

of subjects into retinal and gravitational aṕplies to the effects of letter tilt as well as of letter position.

As in Experiment 1, left and right head-tilt appeared to have different effects on the proportions of gravitation and retinal subjects, but here the difference was in the opposite direction; those who tilted their heads to the left were the more likely to be classified as retinal. Once again, however, the contingency was not significant according to Fisher's exact test $(p=.127)$.

Laterality effects. When the subjects viewed with their heads upright, the position effect broke down into a significant right-field advantage $[F(2,80)=$ 8.23], a significant effect of elevation $[F(2,80)=$ 41.78], and a negligible interaction $[F(2,80)<1]$. The experiment was therefore successful in eliciting a right-field advantage. It was of particular interest, then, to determine whether the right-field advantage would be maintained when the subjects had their heads tilted, and if so, whether it was manifest in retinal or in gravitational coordinates. In fact, the difference between fields was not significant under the head-tilt conditions when positions was defined gravitationally $[\mathrm{F}(1,80)=.54]$ but was highly significant when position was measured retinally $[F(1,80)=50.51]$. Thus it appears that, for the group as a whole, the right-field advantage was manifest in retinal rather than in gravitational coordinates.

In an attempt to determine whether the rightfield advantage was gravitational or retinal among the nine subjects elassified as "gravitational," we computed an analysis of variance for these subjects only, and broke down the position effect into components. On gravitational coordinates, the rightfield advantage was significant $[\mathrm{F}(1,40)=7.48]$. However, there were no significant interactions between fields and elevations $[\mathrm{F}(2,40=.38]$, fields and head tilts $[F(1,40)=1.63]$, or fields, elevations, and head tilts $[F(2,40)=.03]$, suggesting that the right-field advantage was approximately constant for each elevation regardless of head tilt. This pattern of results suggests that the right-field advantage was gravitational rather than retinal, for had it been retinal one would have expected these interactions to be nontrivial.

On retinal coordinates, by contrast, there were significant interactions between fields and elevations 
$[F(2,40)=3.44]$, fields and head tilts $[F(1,40)=$ 9.23], and fields, elevations, and head tilts $[\mathrm{F}(2,40)$ $=4.35]$. However, the right-field advantage was itself highly significant $[F(1,40)=32.37]$ and larger when the subjects tilted their heads than when their heads were upright. This can be attributed at least in part to an artifact. Among this group, under the head-upright condition, the bottom two letters were reported more accurately than the top two (4.87 and 2.89 correct, respectively). Since seven of the nine subjects tilted their heads to the right, and assuming the difference in report of top and bottom letters to be a gravitational rather than a retinal effect, tilting the head would have brought the bottom letters into the right retinal field for the majority of subjects, thus enhancing the right-field advantage on retinal coordinates. Thus, the pattern of field differences in retinal coordinates, including the overall right-field advantage, is consistent with the view that both the laterality effect and the effect of elevation are fundamentally gravitational rather than retinal. That is, visual laterality cannot be related exclusively to the anatomy of the visual system.

\section{Discussion}

The results differed in two important ways from those of Experiment 1. First, the effect of replacing the rectangular surround with a circular one was to alter the pattern of position effects when the subjects viewed with their heads tilted. Thus, for the majority of subjects, position effects were manifest in retinal rather than in gravitational coordinates, although there was clear evidence for a gravitational influence as well. The effect of letter tilt was also more pronounced on gravitational than on retinal coordinates, which is again the opposite of what occurred in Experiment 1. It is likely that the influence of the rectangular surround in Experiment 1 was not to create low-level masking or metacontrast effects, but rather to establish a basis for the subjective reference frame. In Experiment 2, subjects were deprived of this visual basis for defining a gravitational reference frame, and were thus more likely to adopt retinal coordinates.

Secondly, the effect of introducing a verbal fixation task was to elicit a right-field advantage in the detection of the letters. This was manifest in retinal coordinates for those subject whose reference frames were closer to the retinal frame, and in gravitational coordinates for those whose references more nearly coincided with the gravitational frame. In both cases, the interaction between fields and elevations was negligible, indicating that the rightfield advantage was present at all three elevations. This is contrary to the finding of Worrall and Coles (1976) that the right-field advantage in detection of tachistoscopically presented letters was restricted to the horizontal meridian; indeed, they found no overall right-field advantage at all for letters projected at different locations around the clockface. The reason for this discrepancy is not clear to us, although it may have to do with the fact that they used a simple fixation point whereas we presented digits at the fixation point.

\section{GENERAL DISCUSSION}

In the two experiments reported here, the accuracy with which subjects were able to identify singly presented, briefly flashed letters depended upon the positions occupied by the letters in the visual field. By examining the effect of head tilt on this effect, however, it was clear that the reference frame within which it was expressed was neither purely gravitational nor purely retinal. Although individuals could be classified according to whether this reference frame was closer to the gravitational or to the retinal frame, the evidence suggested that even in individual cases it generally lay somewhere in between. In Experiment 2, at least, the reference frame underlying position effects was at least roughly consistent with that underlying letter tilt effects. The simplest interpretation seems to be that the reference frame within which these effects are manifest is, in fact, the subjective reference frame adopted by the subject, i.e., that reference frame which defines for the subject what is upright and what is tilted.

There is perhaps one difficulty with this view, though: Several authors (e.g., Attneave \& Reid, 1968; Olson \& Attneave, 1970) have shown that even when observers adopt a retinal frame of reference, they remain aware of the gravitational vertical. We suggest, however, that there is a distinction between the subjective reference frame underlying the perception of figural information and one's knowledge of the direction of gravity. The influence of the subjective reference frame may not be all-pervasive; one may interpret the world in gravitational coordinates while remaining aware that one's head is tilted, and by how much, just as one may interpret the world from a retinal perspective while remaining aware of the direction of gravity.

In the introduction, we stressed the role of the stimuli themselves in determining the location of the subjective reference frame. The present experiments make it clear that quite subtle task factors may also exert a profound influence. For instance, the shape of the visual surround appears to have had a decisive effect; in Experiment 1, where the surround was rectangular on gravitational coordinates, the subjective reference frame generally lay closer to the gravitational than to the retinal frame, but this was 
reversed in Experiment 2 where the surround was circular. Overall, the reference frame lay closer to the retinal frame than in studies on the mental rotation of alphanumeric characters (Corballis et al., 1976; Corballis et al., 1978). Where the principal constraint is on detection, there may be a bias toward retinal interpretation, compared to the case where the constraint is one of time. But the important finding from our experiment is that there are marked individual differences in the choice of reference frame, emphasizing again that the reference frame is essentially a subjective one.

Our results make it seem unlikely that the position and orientation of each letter were first perceptually registered on retinal coordinates and then corrected to conform to the subjective reference frame, as Rock (1973) proposed. There is little time for correction to occur prior to the identification of a tachistoscopically presented letter. It is more likely that the perceptual system is pretuned to a particular subjective reference frame, and that retinal information is incorporated directly into that frame. Such a system would maintain the smooth flux of perception in everyday life despite the regular occurrence of head and body tilts. According to this view, the retina is best conceived as providing for the pick-up of visual information for direct incorporation into an ongoing perceptual schema, rather than as a device for forming an image which must then be corrected to form a percept (cf. Neisser, 1976).

It is of some interest that the subjective reference frame seldom coincided with the gravitational one, even in Experiment 1, where the stimulus surround provided continuous information as to the horizontal and vertical axes in gravitational coordinates. We find this surprising, given that it is generaly advantageous in everyday experience to maintain a gravitational framework. It is unlikely that the lack of correspondence between the subjective and gravitational frameworks was due simply to the Aubert effect (Aubert, 1861; Day \& Wade, 1969), which is the small constant error tilted subjects make in attempting to set a luminous rod to the vertical in an otherwise dark room. For head and body tilts of up to about $60^{\circ}-80^{\circ}$, the apparent vertical is misplaced a few degrees from the true vertical in the direction opposite to the tilt of the subject, and it is only beyond this range of tilts that the error is in the same direction as the tilt of the subject (Bauermeister, 1964; Day \& Wade, 1969). With the head tilted $60^{\circ}$, as in our experiments, the effect would be very slight and, if anything, in the direction opposite to that which prevailed in our experiments.

It is possible that the subjects wavered between a gravitational and a retinal framework, and that our results represent the averaging of these states. Our experiments lacked the grain necessary to distinguish this possibility from the alternative one that the subjective reference frame can vary in continuous, analogue fashion from one angular location to another. The latter, more flexible scheme seems the more likely, given that we sometimes operate within environments which are tilted with respect to both gravity and the axes of our heads, as in a tilted vehicle or in reading a tilted page. In gur experiments, there was little advantage to be gained by exclusively adopting either a gravitational or a retinal reference frame, except that it is surprising that the choice of frame did not appear to be influenced by the tilt of the letters, given that the letters always appeared at the same tilt within each testing session; rather the accuracy with which the letters were detected seemed to be influenced by the choice of the subjective reference frame. The angular location of the subjective reference frame may have been determined simply by an averaging of the conflicting cues. Thus there were cues from the gravity receptors, from the contact of the head with the tachistoscope, from contact with the floor, chair, and table on which the tachistoscope was placed, and so on. In Experiment 1, the rectangular surround provided an additional gravitational cue. These various cues may have been weighted differently in different subjects, or there may have been an element of choice, giving rise to individual differences. In experiments on the mental rotation of alphanumeric characters, Corballis et al. (1978) also observed that the subjective reference frame typically lay between the gravitation and retinal frames. Subjects were able to manipulate the location of the subjective frame in response to instructions, but to a rather limited extent.

Insofar as the overall effect of position was manifest on subjective rather than retinal coordinates, one might suppose that this was also true of the difference between visual fields. More exacting tests of the source of laterality effects proved difficult to make, however. In Experiment 1, there was no reliable overall difference between the visual fields when subjects viewed with their heads upright, so there seemed no way to determine from the grouped data whether field differences were retinal or perceptual under the head-tilt condition. However, there was a higher correlation between field differences under the head-upright conditon and those under the head-tilt condition when the latter were measured on gravitational coordinates than when they were measured on retinal coordinates. Although this difference was not statistically significant, it does suggest that the laterality effect is perceptual rather than retinal, since the subjective reference frame generally lay closer to the gravitational than to the 
retinal reference frame in this experiment. In Experiment 2, there was a reliable overall right-field advantage when the subjects viewed with their heads upright, but in this experiment the subjective reference frame generally lay closer to retinal than to the gravitational frame, making it difficult to distinguish the perceptual from the retinal. However, among the minority of subjects who evidently adopted a reference frame that was more gravitational than retinal, the pattern of field differences generally conformed better to a gravitational than to a retinal interpretation. Thus, the data generally suggest that lateral asymmetry in tachistoscopic perception can be decoupled from the anatomy of the retinocortical projections, and is thus truly perceptual rather than anatomical.

\section{REFERENCES}

Attneave, F., \& Olson, R. Discriminability of stimuli varying in physical and retinal orientation. Journal of Experimental Psychology, 1967, 74, 149-157.

Atrneave, F.. \& Reid, K. Voluntary control of frame of reference and shape equivalence under head rotation. Journal of Experimental Psychology, 1968, 78, 153-159.

Aubert, H. Eine scheinbare bedeutende Drehung con Objekten bei Neigung des Kopfes nach rechts oder links. Virchows Archiv für Pathologische Anatomie und Physiologie und für Klinische Medizin, 1861, 20, 381-393.

Bauermeister, M. The effects of body tilt on apparent verticality, apparent body position, and their relation. Journal of Experimental Psychology, 1964, 67, 142-147.

BRYDEN. M. P. Tachistoscopic recognition, handed ness and cerebral dominance. Neuropsychologia, 1965, 3, 1-8.

Cooper, L. A., \& Shepard, R. N. Chronometric studies of the rotation of mental images. In W. G. Chase (Ed.), Visual information processing. New York: Academic Press, 1973.

Corballis, M. C., Nagourney, B. A., Shetzer, L. I., \& Stefanatos, G. Mental rotation under head tilt: Factors influencing the location of the subjective reference frame. Perception \& Psychophysics, 1978, 24, 263-273.

Corballis, M. C., \& Roldan, C. E. Detection of symmetry as a function of angular orientation. Journal of Experimental Psychology: Human Perception \& Performance, 1975, 1, 221-230.

Corballis, M. C., Zbrodoff, J., \& Roldan, C. E. What's up in mental rotation? Perception \& Psychophysics, 1976, 19. $525-530$.

Davidson, M. L., Fox, M. J., \& Dick, A. D. Effects of eye movements on backward masking and perceived location. Perception \& Psychophysics, 1973, 14, 110-116.

DAY, R. H., \& WADE, N. J. Mechanisms involved in visual orientation constancy. Psychological Bulletin, 1969, 71, 33-42.

Geffen, G., Bradshaw, J. L., \& Wallace, G. Interhemispheric effects on reaction time to verbal and nonverbal stimuli. Journal of Experimental Psychology, 1971, 87, 415-422.

GiBson, J. J. The senses considered as perceptual systems. Boston: Houghton Mifflin, 1966.

HELlige. J. B. Visual laterality patterns for pure- versus mixed-list presentations. Journal of Experimental Psychology: Human Perception and Performance, 1978, 4, 121-131.
Kershner, J., Thomae, R., \& Callaway, R. Nonverbal fixation control in young children induces a left-field advantage in digit recall. Neuropsychologia, 1977, 15, 569-576.

KimURA, D. Cerebral dominance and the perception of verbal stimuli. Canadian Journal of Psychology, 1961, 15, 166-171.

Kimura, D. Dual functional asymmetry of the brain in visual perception. Neuropsychologia, 1966, 4, 275-285.

KImURA, D. Functional asymmetry of the brain in dichotic listening. Cortex, 1967, 3, 163-178.

KIMURA, D. Spatial localization in left and right visual fields. Canadian Journal of Psychology, 1969, 23, 445-458.

KinsBouRnE, $M$. The control of attention by interaction between the hemispheres. In S. Kornblum (Ed.), Attention and performance. IV. New York: Academic Press, 1973.

Kinsbourne, M. The mechanism of hemispheric control of the lateral gradient of attention. In P. M. A. Rabbitt \& S. Dornic (Eds.), Attention and performance V. New York: Academic Press, 1975.

Mrller, E. F. Counterrolling of the human eyes produced by head tilt with respect to gravity. Acta Oto-laryngologica, $1962,54,479-501$.

Mishkin, M., \& Forgays, D. G. Word recognition as a function of retinal locus. Journal of Experimental Psychology, 1952, 43, 43-48.

Morais, J., \& Bertelson, P. Spatial position versus ear of entry as determinant of the auditory laterality effects. Journal of Experimental Psychology, 1975, 1, 253-262.

Neisser, U. Cognition and reality. San Francisco: Freeman, 1976.

Olson, R. K., \& Attneave, F. What variables produce similarity grouping? American Journal of Psychology, 1970, 83, 1-21.

Rock, I. Orientation and form. New York: Academic Press, 1973.

Rock, I., \& Heimer, W. The effect of retinal and phenomal orientation on the perception of form. American Journal of Psychology, 1957, 70, 493-511.

Rock, I., \& Leaman, R. An experimental analysis of visual symmetry. Acta Psychologica, 1963, 21, 171-183.

TURVEY, M. T. Contrasting orientations to the theory of visual information processing. Psychological Review, 1977, 84, 67-88.

Vaughan, G. M., \& Corballis, M. C. Beyond tests of significance: Estimating strength of effects in selected ANOVA designs. Psychological Bulletin, 1969, 72, 204-213.

Whire, C. W. Visual masking during pursuit eye movements. Journal of Experimental Psychology: Human Perception \& Performance, 1976, 2, 469-478.

WhITE, M. J. Laterality differences in perception: A review. Psychological Bulletin, 1969, 72, 387-405.

WINER, B. J. Statistical principles in experimental design. New York: McGraw-Hill, 1971.

Worrall, N., \& Coles, P. Visual field differences in recognizing letters. Perception \& Psychophysics, 1976, 20, 21-24.

\section{NOTE}

1. The significance level used throughout is $p<.05$.

(Received for publication August 16, 1977; revision accepted May 24, 1978.) 